\title{
PROCESOS ACTUALES Y PERSPECTIVAS PARA EL TRANSPORTE FERROVIARIO DE VIAJEROS EN ANDALUCÍA
}

\author{
Antonio Gavira Narváez y Jesús Ventura Fernández \\ Departamento de Geografía Física y Análisis Geográfico Regional \\ Universidad de Sevilla
}

\section{RESUMEN}

El papel que juega el ferrocarril en los sistemas urbanos e interurbanos de transporte, gracias al desarrollo técnico y organizativo, ha impulsado que tenga una participación cada vez más relevante en el tráfico especializado de viajeros en Andalucía. Además, su integración con otros medios de desplazamiento viabiliza su uso y competitividad.

A pesar de la gran presencia que ha adquirido el medio, su estudio resulta novedoso y de gran actualidad, ya que ha sido bastante menos tratado, con respecto a otros sistemas de transporte, dentro de los trabajos científicos a nivel español y andaluz. La elección de la escala regional para analizar la red ferroviaria se fundamenta en tres factores, como son la extensión, la demografía y la localización de Andalucía. Éstos le confieren una identidad histórica y cultural propia, así como elementos suficientes para analizar la región de forma individualizada dentro del entramado ferroviario español.

Además, la descentralización de algunas políticas de transporte le concede mayor consistencia al estudio realizado. La elección del ámbito andaluz pretende, por tanto, abordar este territorio como un espacio concreto de España y del conjunto de la Unión Europea.

Palabras clave: ferrocarril, movilidad, intermodalidad, sostenibilidad, SIG-T, transporte, redes, infraestructuras.

\section{ABSTRACT}

\section{Current processes and perspectives for the rail travellers transport in Andalusia}

The role of the railway systems in urban and interurban transport, thanks to the technical and organizational development, has been driven an ever more relevant in specialized traveler traffic in Andalusia. In addition, its integration with other means of travel makes viable its use and competitiveness.

Despite the strong presence it has acquired the environment, their study is novel and very timely, as it has been much less treated, with respect to other transport systems within the scientific work at the Spanish and Andalusian level. The choice of regional scale for analyzing rail network is based on three factors, including the size, location and demographics of Andalusia. They give it a historical and cultural identity of its own, and sufficient elements to analyze individually the region within the Spanish railway network.

In addition, the decentralization of some transport policies gives more consistency to the study. The choice of the region of Andalusia intends therefore, address this particular territory as a specific space within Spain and the whole European Union.

Key words: railroad, mobility, intermodality, sustainability, GIS-T, transport, networks, infrastructures. 


\section{INTRODUCCIÓN}

El transporte ferroviario de viajeros ha adquirido en los últimos tiempos una progresiva importancia, convirtiéndose en un elemento esencial en la movilidad cotidiana de distintos ámbitos urbanos de Andalucía, como los de Cádiz, Málaga y Sevilla. Gracias a ello, se ha ampliado la presencia del metro ligero, así como las líneas de alta velocidad para los desplazamientos entre las grandes urbes andaluzas. La relevancia alcanzada es consecuencia de los cambios que se han producido en los desplazamientos que realiza la población, destacando entre ellos los motivos laborales y el acceso a distintos bienes y servicios (Gavira Narváez, 2008). Esto ha propiciado que en la actualidad se intente mejorar su organización, gestión y optimización (Seguí Pons y Martínez Reynés, 2004).

La aparición de los transportes urbanos, entre ellos el ferroviario, surgió paralelamente al desarrollo de la ciudad industrial, cuando se conforma el territorio ajustado a las necesidades de consumo y producción (Miralles-Guasch, 2002). Estos cambios propiciaron nuevas demandas de desplazamiento más veloces y adecuadas para una población que, si en un primer momento trabajaba y vivía en espacios adyacentes, con el tiempo terminará haciéndolo en territorios distantes, debido a la expansión urbana extramuros.

Éstas son las principales secuelas del nuevo modelo de crecimiento, consolidado desde mediados del siglo XX. El hecho de tener que trabajar en otra localidad no sólo supone el condicionante de desplazarse de forma cotidiana, sino la necesidad de recurrir a algún medio de transporte. Éste suele ser generalmente, y en la actualidad, el vehículo privado, que es utilizado de manera indiscriminada para estos viajes, así como para distancias medias dentro del mismo municipio.

El uso extendido del automóvil es la principal consecuencia de la escasez de medios públicos de calidad que puedan absorber estos desplazamientos. Este modelo de crecimiento se ha traducido en una pérdida constante de la calidad de vida de los ciudadanos, que observan cómo, con el paso de los años, tardan más tiempo en recorrer distancias similares en las horas puntas de tráfico. Además, el uso masivo del automóvil ha propiciado, entre otros inconvenientes, el aumento de la contaminación, de enfermedades relacionadas con la polución y el ruido, segregación social, o deterioro de edificios singulares.

La principal medida para mitigar esta situación ha sido la creación de alternativas al vehículo privado que hagan atractivo su uso en tiempo, periodicidad, comodidad y precio. Éstas han pasado, irremediablemente, por la potenciación de los transportes públicos, centrados en los ferroviarios, pues facilitan la canalización de la movilidad urbana e interurbana, completada con otros medios como el autobús, la bicicleta, o el ir a pie.

\section{EVOLUCIÓN DEL FERROCARRIL}

Los antecedentes del ferrocarril en España vienen marcados por la construcción tardía de las primeras líneas, a pesar de existir algunos ensayos coetáneos a los movimientos británicos iniciales. Las primeras concesiones se formalizaron en Andalucía, para construir los trazados entre Jerez de la Frontera y El Portal en 1829, así como entre Jerez y El Puerto de Santa María, Rota y Sanlúcar de Barrameda en 1830 (Consejería de Obras Públicas y Transportes, 1988). Sin embargo estos proyectos fracasaron por falta de capital. El ferrocarril no empezó a recorrer la geografía ibérica hasta que fue aprobado el permiso solicitado por José María Roca en 1843, inaugurándose en 1848 el trazado entre Barcelona y Mataró.

Las debilidades actuales del transporte ferroviario se pueden achacar a la elección de ancho de vía, así como a la construcción de una red radial, lo que ha marcado la versatilidad del entramado español y andaluz (López Pérez, 1990). Esta decisión ha podido condicionar, al menos en parte, el desarrollo de regiones periféricas como la andaluza, pues la disposición de redes radiales, tanto para las carreteras como para el ferrocarril, provocó que las conexiones interprovinciales fuesen muy difíciles.

El perfeccionamiento de los medios de transporte mecánicos, principalmente el ferrocarril en el siglo XIX, trajo consigo la incorporación del tren y el tranvía al trazado de muchas ciudades, como en Sevilla, Cádiz o Málaga. Esto determinó la morfología y la funcionalidad urbana, pues desplazó a las viejas diligencias y carricoches. 
La construcción de la incipiente red ferroviaria y de sus estaciones no desaprovechó la oportunidad de emplazarlas en núcleos donde pudiesen atraer viajeros y mercancías, lo que favoreció que la mayoría de los núcleos andaluces más poblados antes de la Guerra Civil dispusieran de estación. El ferrocarril puso las bases de las transformaciones urbanísticas de estas ciudades, convirtiéndose las estaciones en elementos polarizadores de su estructura, paisaje y percepción.

Los inversores que apostaron por el medio buscaron que la rentabilidad fuese rápida. La red ferroviaria se extendió vertiginosamente por gran parte de Andalucía, superando el centenar los municipios que han tenido servicios ferroviarios o tranviarios. Por ello, además de los aspectos demográficos, se buscó que las nuevas estaciones cumpliesen alguno de estos factores (Vega González, 1990):

- Grandes ciudades: El emplazamiento de las nuevas estaciones será un factor decisivo para la extensión y uso óptimo de la red ferroviaria. Por ello, los cinco núcleos andaluces más poblados (Sevilla, Málaga, Granada, Córdoba y Cádiz) contarán con servicios ferroviarios para dar salida a los productos de sus respectivas áreas de influencia. Además, el disponer de un volumen de usuarios considerable hizo factible las fuertes inversiones realizadas en la construcción y puesta en funcionamiento de las infraestructuras.

- Núcleos agrarios o mineros: El sector primario a finales del siglo XIX y principios del XX era parte esencial de la economía española en general y andaluza en particular. Sin embargo, los sistemas de distribución de los recursos, tanto dentro como fuera de la región, necesitaban mejoras que optimizasen su traslado. Esta premisa ayudará a emplazar estaciones en localidades como Marchena, Puente Genil, Úbeda, Torredonjimeno o Antequera.

- Grandes puertos: El ferrocarril desde su nacimiento intentó interrelacionarse con otros medios de transporte como el marítimo. La potencialidad que suponía dar salida a las materias primas de los núcleos interiores impulsó la planificación de trazados dirigidos a los puertos principales, como los de Cádiz, Málaga, Algeciras o Almería.

La rápida implantación del ferrocarril en Andalucía durante el siglo XIX propició la articulación del extenso territorio andaluz (más de $87.000 \mathrm{~km} 2$ ) en pocas décadas. No obstante, la escasa planificación, el pobre mantenimiento de las infraestructuras, y la aparición del automóvil con el consiguiente descenso paulatino de la demanda, provocó que el medio fuese languideciendo hasta el nuevo impulso que se viene realizando desde finales del siglo XX.

Asimismo, el crecimiento de la red ferroviaria y la implantación en el medio urbano de sus infraestructuras produjo efectos diversos que en algunos casos no han sido solucionados y que fueron originados por las apetencias de los capitales privados. Éstos ocasionaron variadas percepciones del medio entre los ciudadanos, que pasaron de ver el ferrocarril como una innovación beneficiosa a una molestia para el crecimiento urbano. Las principales consecuencias fueron las siguientes:

- Creación de estaciones alejadas de los núcleos urbanos, que generaron nuevas localidades a su alrededor, como la de Bobadilla, en el término municipal de Antequera.

- Segregación en ciudades por la trama ferroviaria, como sufrió, hasta la década de los noventa del pasado siglo XX, la propia ciudad de Sevilla.

- Inconvenientes ocasionados por la construcción de más de una estación férrea, así como el efecto barrera que imponían para el crecimiento urbano, casos por ejemplo de Sevilla o Huelva.

El actual estado de las autonomías ha favorecido el impulso y la modernización de la red ferroviaria andaluza gracias a los acuerdos establecidos entre la Junta de Andalucía, RENFE y ADIF. Esta nueva situación fue refrendada con la construcción de la primera línea de alta velocidad entre Madrid y Sevilla por parte del gobierno central (1992), dotando a la red de un halo de modernidad y utilidad que había ido perdiendo durante el régimen anterior. Además, su construcción implicó un cambio de mentalidad que ha impregnado a la sociedad y a las administraciones que gestionan en la actualidad el entramado ferroviario. 
Sin embargo, las intervenciones emprendidas están condicionadas por las decisiones tomadas durante la conformación inicial de la red, que en su mayoría fueron poco afortunadas (Carbonell Romero, 1990); y el resultado fundamental ha sido la fuerte inversión en la construcción y adecuación de las infraestructuras viarias para tráfico por carretera, y en una ínfima para las ferroviarias.

Además, las secuelas se han dejado notar en el papel secundario que ocupa el ferrocarril en la mayoría de las regiones españolas, excepto en aquellas capitales de provincia que han generado extensas aglomeraciones, ya que en ellas los problemas de tráfico han potenciado la consolidación de las redes de transporte ferroviario urbano e interurbano.

\section{CONTEXTUALIZACIÓN DEL FERROCARRIL EN ANDALUCÍA}

El futuro de la red andaluza es esperanzador, pues documentos como el Plan de Infraestructuras para la Sostenibilidad del Transporte en Andalucía (2007-2013) convierten a los diferentes modos ferroviarios en los ejes del transporte colectivo en la Comunidad. Las principales actuaciones que proponen se decantan por la extensión de los servicios de cercanías a todos los llamados centros regionales, el fomento de los ferrocarriles metropolitanos (como el metro o el tranvía), la ampliación de la red de alta velocidad, y la modernización de los trazados que prestan servicios de media distancia.

Sin embargo, existen espacios que no son atendidos directamente, y que debido a su peso demográfico, resulta aconsejable su inclusión dentro del entramado ferroviario regional (mapa 1). El mapa ferroviario planificado gravita en torno a la extensión de la red de alta velocidad, la mejora e incremento de los núcleos de cercanías, y la construcción de ferrocarriles metropolitanos (metro, tranvía y cercanías). Estas actuaciones se extienden por casi toda la geografía andaluza, no relegando a ninguna provincia.

La prolongación del entramado de alta velocidad mejorará las conexiones entre las capitales de los denominados por el Plan de Ordenación del Territorio de Andalucía (Pota, 2006) centros regionales andaluces, así como con las comunidades autónomas colindantes y con el centro de España. En la actualidad, la red AVE llega sólo a las ciudades de Córdoba, Málaga y Sevilla, aunque son varios los proyectos que están en marcha y que culminarán con su extensión por el conjunto andaluz.

De manera complementaria, en estos ámbitos se ha impulsado la creación de numerosas iniciativas que tienen como fin mejorar o crear nuevas redes de ferrocarriles metropolitanos, debido a la congestión de las principales arterias urbanas y metropolitanas en los grandes núcleos andaluces. Las infraestructuras que se encuentran en mayor grado de planificación o desarrollo son las siguientes:

- Cercanías: En los próximos años se pretenden fortalecer los servicios actuales en los ámbitos de Cádiz, Málaga y Sevilla. Del mismo modo, está prevista la creación de nuevas redes, entre otras, en Córdoba y Jaén. La transferencia de las competencias sobre los servicios de cercanías que marca el Estatuto de Autonomía (2007), y su extensión a todas las aglomeraciones urbanas, recogida en el Plan de Infraestructuras para la Sostenibilidad del Transporte en Andalucía (PISTA 2007-2013), son los principales desafíos que tendrán lugar en los próximos años.

- Metro: La finalización de los proyectos en construcción en Granada, Málaga y Sevilla será la actuación esencial que se efectuará en los próximos años. Su culminación generará nuevas pautas de movilidad en la población beneficiaria, como ya ocurre, aunque aún de forma muy parcial, con la línea 1 del metro de Sevilla. No obstante, los problemas de financiación, originados por la crisis económica iniciada en 2008, va a retrasar la construcción del trazado planificado restante en Sevilla, de forma que el debate inicial entre la ampliación en ramales de la única línea actual o la ejecución de nuevas líneas completas $(2,3$ y 4$)$ ha quedado aplazado.

- Tranvía: Los nuevos proyectos tranviarios han renacido en las principales ciudades andaluzas, a pesar de la rapidez con la que fueron desmanteladas las antiguas infraestructuras desde mediados del siglo XX. En estos momentos, sólo existe una línea en servicio: en el año 2007 se inauguró el tranvía urbano de Sevilla, denominado Metrocentro, debido a su localización en el centro histórico de la ciudad. 


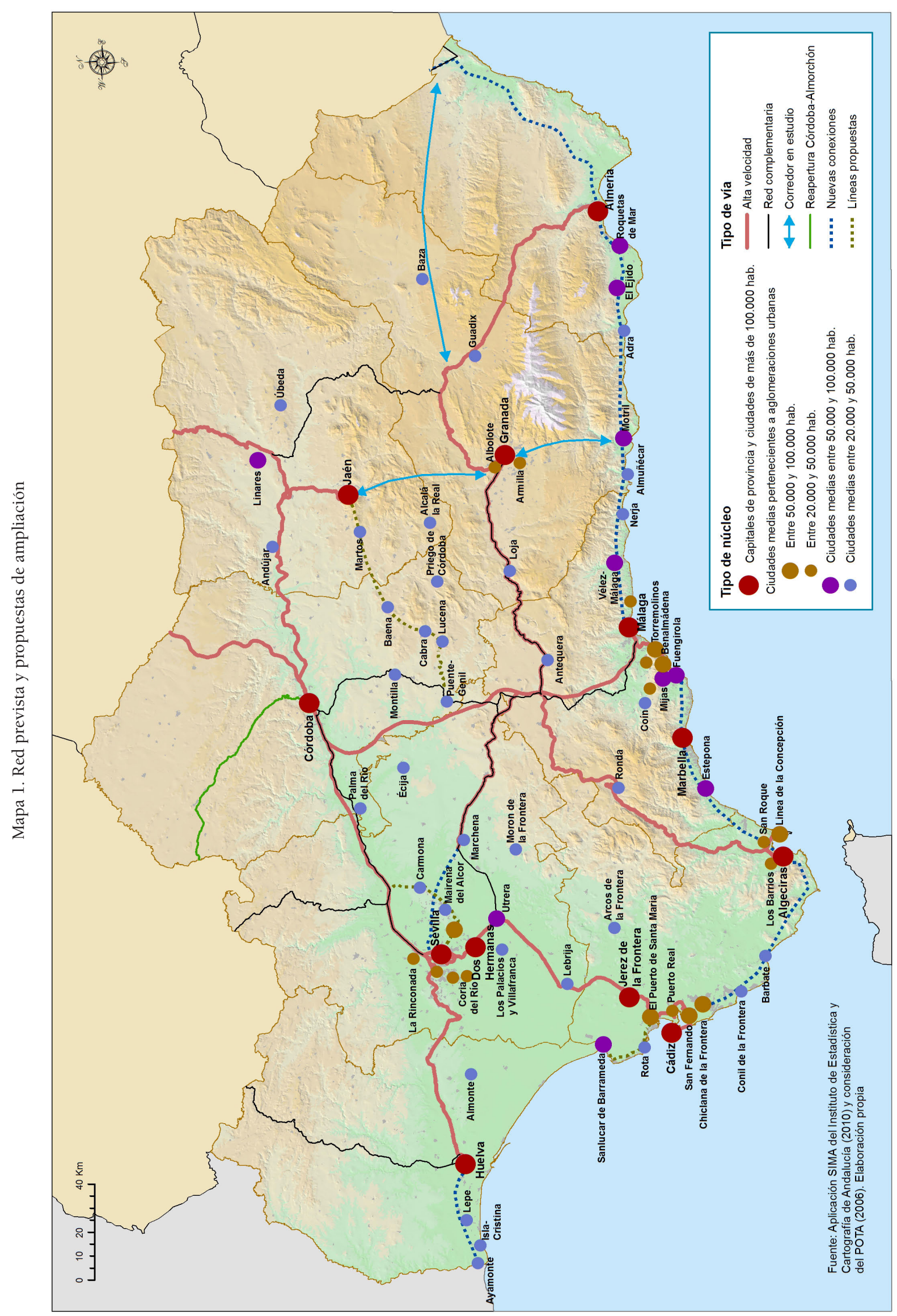


A esta línea hay que añadir diversas actuaciones con un grado de ejecución desigual, siendo algunos meros proyectos, como los de Huelva o Almería. En el caso del trazado entre Vélez-Málaga y Torre del Mar, abierta en 2006, éste se encuentra clausurado por baja afluencia de usuarios debido a la inadecuada localización de los apeaderos terminales de la línea. En construcción se pueden señalar los tranvías de Bahía de Cádiz, Alcalá de Guadaíra o Dos Hermanas. Asimismo, es preocupante resaltar cómo, en la ciudad de Jaén, y a pesar de haberse finalizado la construcción de una línea en mayo de 2011, no existe en la actualidad empresa concesionaria que preste los servicios, debido a su previsible falta de rentabilidad.

El potencial de los trazados ferroviarios va más allá de su uso, ya que incluso, cuando han sido desmanteladas las infraestructuras por la pérdida de viabilidad económica, se han reconvertido en algunos casos en Vías Verdes (Aycart Luengo, 2001). Éstas son recorridos idóneos para practicar actividades lúdicas, debido a sus suaves pendientes y a sus curvas abiertas, heredadas de las exigencias constructivas del primitivo trazado ferroviario.

\section{SITUACIÓN ACTUAL DEL TRANSPORTE FERROVIARIO}

En los últimos años el tren se ha convertido en una alternativa para la realización de los viajes cotidianos y de ocio. No obstante, la red ferroviaria andaluza ha padecido, desde su configuración inicial, carente en muchos casos de planificación, un escaso mantenimiento y renovación, que ha ocasionado que algunos de los servicios sean claramente deficitarios por su reducida calidad y adecuación a la demanda (Consejería de Obras Públicas y Transportes, 1988). Esta situación se ha unido a la escasa competitividad que demuestra el transporte público para resolver los desplazamientos cotidianos de la población andaluza, según se observa en la tabla 1 , con una representatividad del ferrocarril del $0,2 \%$ y de los autobuses urbanos e interurbanos del 1,4\%.

Tabla 1. Desplazamientos según modo principal de transporte por provincia andaluza en día laborable (valores absolutos en miles), 2006.

\begin{tabular}{|c|c|c|c|c|c|c|c|}
\hline & \multicolumn{6}{|c|}{ Modo principal de transporte } & \multirow[b]{2}{*}{ Total } \\
\hline & $\begin{array}{l}\text { Más } 5 \text { min. a } \\
\text { pie o bicicleta }\end{array}$ & Coche o moto & $\begin{array}{l}\text { Autobús } \\
\text { urbano }\end{array}$ & $\begin{array}{c}\text { Autobús } \\
\text { interurbano }\end{array}$ & Tren & Otros & \\
\hline Provincia & Desplaz. (\%) & Desplaz. (\%) & Desplaz. (\%) & Desplaz. (\%) & Desplaz. (\%) & Desplaz. (\%) & Desplaz. \\
\hline Almería & $555,0(39,1)$ & $783,6(55,2)$ & $19,2(1,4)$ & $22,4(1,6)$ & $1,3(0,1)$ & $37,9(2,7)$ & $1.419,4$ \\
\hline Cádiz & $1.726,2(46,6)$ & $1.759,4(47,5)$ & $111,5(3,0)$ & $36,0(1,0)$ & $17,4(0,5)$ & $51,6(1,4)$ & $3.702,1$ \\
\hline Córdoba & $1.079,2(51,9)$ & $870,2(41,8)$ & $52,9(2,5)$ & $33,1(1,6)$ & $2,4(0,1)$ & $42,6(2,0)$ & $2.080,3$ \\
\hline Granada & $1.058,1(48,6)$ & $944,2(43,4)$ & $79,0(3,6)$ & $50,7(2,3)$ & $0,0(0,0)$ & $43,9(2,0)$ & $2.175,9$ \\
\hline Huelva & $617,8(51,1)$ & $535,0(44,2)$ & $16,2(1,3)$ & $17,9(1,5)$ & $0,8(0,1)$ & $21,8(1,8)$ & $1.209,5$ \\
\hline Jaén & $770,6(51,9)$ & $607,9(40,9)$ & $57,6(3,9)$ & $18,1(1,2)$ & $0,0(0,0)$ & $31,4(2,1)$ & $1.485,7$ \\
\hline Málaga & $1.430,5(47,6)$ & $1.290,8(43,0)$ & $183,0(6,1)$ & $29,1(1,0)$ & $14,1(0,5)$ & $56,9(1,9)$ & $3.004,4$ \\
\hline Sevilla & $1.807,0(37,5)$ & $2.606,8(54,1)$ & $248,3(5,2)$ & $76,6(1,6)$ & $11,7(0,2)$ & $65,0(1,4)$ & $4.815,5$ \\
\hline Total & $\begin{array}{c}9.044,4 \\
(45,5)\end{array}$ & $\begin{array}{c}9.397,9 \\
(47,2)\end{array}$ & $767,7(3,9)$ & $283,9(1,4)$ & $47,7(0,2)$ & $351,1(1,8)$ & $19.892,8$ \\
\hline
\end{tabular}

Fuente: Ministerio de Fomento MOVILIA 2006. Elaboración propia 
El uso del ferrocarril llega a ser casi nulo en las provincias de Granada y Jaén. El coche o la motocicleta son los medios más utilizados para realizar los desplazamientos cotidianos, con cifras que rondan el 50\%, seguido de aquéllos realizados a pie o en bicicleta.

Las relaciones ferroviarias entre las capitales andaluzas se caracterizan, en general, por no realizarse a través de servicios directos, sino a partir de transbordos que suelen dilatar en demasía los tiempos de viaje. Solamente Sevilla dispone de conexiones directas con todas las capitales de provincia, lo que se traslada a unos plazos más competitivos, como se observa en la tabla 2. Otras, como Huelva, únicamente poseen servicios directos con Sevilla, condicionando sus enlaces a los disponibles en la capital andaluza.

La ciudad de Málaga sólo dispone de servicios directos con Córdoba y Sevilla, a pesar de ser el segundo núcleo más poblado del Sur de España. En los casos de Granada y Almería disponen de servicios directos entre ellas y con Sevilla, así como de un enlace directo entre Granada y Córdoba, según se observa en la referida tabla 2.

Tabla 2. Tiempo de recorrido en ferrocarril entre las capitales andaluzas (en horas y minutos), 2009.

\begin{tabular}{|c|c|c|c|c|c|c|c|c|}
\hline & Almería & Cádiz & Córdoba & Granada & Huelva & Jaén & Málaga & Sevilla \\
\hline Almería & & $7: 43$ & $5: 22$ & $2: 04^{\mathrm{a}}$ & $7: 38$ & $4: 29$ & $4: 47$ & $5: 05^{\mathrm{a}}$ \\
\hline Cádiz & $7: 15$ & & $2: 35^{\mathrm{a}}$ & $4: 54$ & $3: 59$ & $4: 13^{\mathrm{a}}$ & $3: 17$ & $1: 40^{\mathrm{a}}$ \\
\hline Córdoba & $4: 55$ & $2: 31^{\mathrm{a}}$ & & $2: 32^{\mathrm{a}}$ & $1: 55^{\mathrm{a}}$ & $1: 36^{\mathrm{a}}$ & $1: 17^{\text {af }}$ & $1: 11^{\mathrm{ab}}$ \\
\hline Granada & $2: 06^{\mathrm{a}}$ & $5: 31$ & $2: 27^{\mathrm{a}}$ & & $4: 40$ & $3: 59$ & $2: 30$ & $2: 57^{\mathrm{a}}$ \\
\hline Huelva & $7: 52$ & $3: 36$ & $1: 52$ & $5: 12$ & & $4: 05$ & $3: 40$ & $1: 32^{\mathrm{a}}$ \\
\hline Jaén & $4: 18$ & $4: 08^{\mathrm{a}}$ & $1: 31^{\mathrm{a}}$ & $3: 52$ & $4: 05$ & & $2: 50$ & $2: 23^{\mathrm{a}}$ \\
\hline Málaga & $4: 35$ & $3: 25$ & $1: 10^{\text {ac }}$ & $2: 20$ & $3: 39$ & $3: 04$ & & $2: 30^{\text {ad }}$ \\
\hline
\end{tabular}

Fuente: Consejería de Obras Públicas y Vivienda - Gerencia de Regionales de Renfe, citado en el Anuario Estadístico de Andalucía 2010. Elaboración propia

Las mejoras emprendidas en la red han favorecido que el número global de viajeros se haya incrementado en los últimos años, como se aprecia en el gráfico 1. Esto ha conducido a un crecimiento que supera los cinco millones de usuarios en el periodo comprendido entre 2001 y 2008, consecuencia del proceso gradual de adecuación y ampliación de los servicios que presta Renfe dentro de la comunidad autónoma de Andalucía. No obstante, en el año 2009, se produce un descenso que ronda los dos millones de viajeros, efecto de la crisis económica, siguiendo la tendencia general a escala nacional.

Gráfico 1. Evolución del número de viajeros en los trenes de renfe en andalucía (millones de viajeros), 2001-2009.

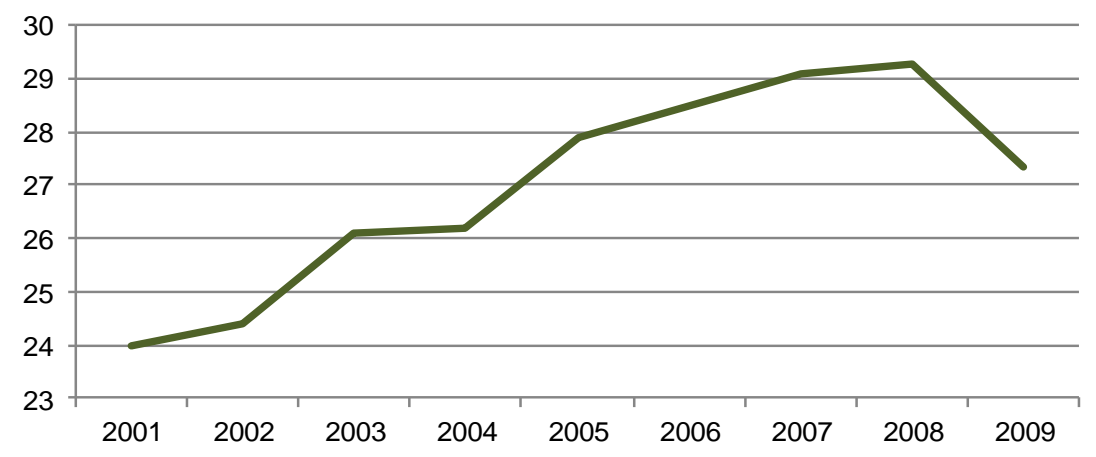

Fuente: Renfe, citado en el Anuario Estadístico de Andalucía 2010. Elaboración propia 
Desafortunadamente, el incremento acontecido en el periodo de bonanza de principios del siglo XXI se ha visto frenado por la crisis económica que le ha seguido desde finales de la primera década, provocando un descenso en el número de usuarios, bastante claro al final del periodo analizado. Éste es el caso de la provincia de Málaga, donde las diferencias rondan el millón de personas. En el periodo comprendido entre 2006 y 2009 se origina una regresión a nivel regional que supera el 4\%. Lo mismo ocurre en las provincias de Cádiz, Málaga y Sevilla, alcanzando la gaditana el 16\%. El resto de territorios ha experimentado crecimientos, entre los que destacan el 8\% de Almería y el 9\% de Córdoba.

A esta problemática se une la heterogénea distribución del entramado ferroviario andaluz, lo que provoca que dentro de la provincia de Sevilla se localice algo más del 20\% de la red de ancho ibérico, mientras que en Almería o Cádiz no se alcanza ni un 10\%. Asimismo, el predominio de las vías simples es abrumador. Las diferencias en las tipologías de las infraestructuras quedan patentes en la existencia solamente de vías dobles electrificadas en las provincias de Cádiz, Málaga y Sevilla. La escasa longitud de las vías dobles impide el incremento y una mayor calidad de los servicios de viajeros, ya que limita las posibilidades de maniobra del tráfico ferroviario. Su extensión no llega a los 150 kilómetros, concentrándose cerca del 70\% en la provincia de Sevilla, como vemos en la tabla 3.

Los trazados de alta velocidad se localizaban en el año 2009 únicamente en las provincias de Córdoba, Sevilla y Málaga, aunque está prevista su extensión por todo el territorio andaluz. Por Córdoba discurren 170,9 kilómetros, el 47,7\% para este tipo de vía, como consecuencia del emplazamiento de los trazados que conducen a Sevilla y a Málaga. Sevilla se mantiene en una posición intermedia, con 114,4 kilómetros, mientras que Málaga se ve afectada por 73,3 kilómetros.

Tabla 3. Red ferroviaria según tipo de vía por provincia (en kms), 2009.

\begin{tabular}{|c|c|c|c|c|c|c|c|c|c|}
\hline & Almería & Cádiz & Córdoba & Granada & Huelva & Jaén & Málaga & Sevilla & And. \\
\hline Sin electrificar \\
\hline Sencilla & $\begin{array}{c}19,8 \\
(2,0 \%)\end{array}$ & $\begin{array}{c}58,4 \\
(5,9 \%)\end{array}$ & $\begin{array}{c}113,1^{\mathrm{a}} \\
(11,5 \%)\end{array}$ & $\begin{array}{c}195,9 \\
(19,9 \%)\end{array}$ & $\begin{array}{c}102,8 \\
(10,4 \%)\end{array}$ & $\begin{array}{c}95,3 \\
(9,7 \%)\end{array}$ & $\begin{array}{c}195,1 \\
(19,8 \%)\end{array}$ & $\begin{array}{c}204,3 \\
(20,7 \%)\end{array}$ & 984,7 \\
\hline Electrificada \\
\hline Sencilla & $\begin{array}{c}72,2 \\
(8,8 \%)\end{array}$ & $\begin{array}{c}44,3 \\
(5,4 \%)\end{array}$ & $\begin{array}{c}212,3 \\
(26,0 \%)\end{array}$ & $21,8^{\mathrm{b}}(2,7 \%)$ & $\begin{array}{c}60,8 \\
(7,4 \%)\end{array}$ & $\begin{array}{c}146 \\
(17,9 \%)\end{array}$ & $\begin{array}{c}107,5 \\
(13,2 \%)\end{array}$ & $\begin{array}{c}151,2 \\
(18,5 \%)\end{array}$ & 816,1 \\
\hline Doble & - & $\begin{array}{c}30,0 \\
(20,9 \%)\end{array}$ & - & - & - & - & $\begin{array}{c}13,3 \\
(9,3 \%)\end{array}$ & $\begin{array}{c}100,3 \\
(69,8 \%)\end{array}$ & 143,6 \\
\hline Total & $\begin{array}{c}92,0 \\
(4,7 \%)\end{array}$ & $\begin{array}{c}132,7 \\
(6,8 \%)\end{array}$ & $\begin{array}{c}325,4 \\
(16,7 \%)\end{array}$ & $\begin{array}{c}217,7 \\
(11,2 \%)\end{array}$ & $\begin{array}{c}163,6 \\
(8,4 \%)\end{array}$ & $\begin{array}{c}241,3 \\
(12,4 \%)\end{array}$ & $\begin{array}{c}315,9 \\
(16,2 \%)\end{array}$ & $\begin{array}{c}455,8 \\
(23,4 \%)\end{array}$ & $1.944,4$ \\
\hline \\
Alta V. \\
\hline
\end{tabular}

Fuente: Consejería de Obras Públicas y Vivienda - INECO-TIFSA, citado en el Anuario Estadístico de Andalucía 2010. Elaboración propia

Por estaciones, gran parte de los viajeros en el año 2007 se solía concentrar en torno a los centros regionales, destacando las tres redes de cercanías (Cádiz, Málaga y Sevilla). Esta dinámica es consecuencia de la oferta laboral y de servicios que se genera dentro de ellas, lo que favorece los desplazamientos interiores. Sin embargo, Andalucía cuenta con los servicios de cercanías menos extensos de España, aunque es uno de los espacios más metropolitanizados (Valenzuela Montes, 2009). Así, las tres redes actuales dejan fuera a ciudades de un tamaño medio con una demanda potencial bastante elevada, como, en el caso de Sevilla, a Alcalá de Guadaíra, o en el de Málaga a Marbella o Estepona. Del mismo modo, destaca la pertenencia de Pulpí (provincia de Almería) al núcleo de cercanías de Murcia, no presentando interrelación alguna con el resto de la red ferroviaria andaluza. En cuanto a otros servicios ferroviarios, sólo Sevilla dispone de metro y tranvía, aunque su extensión es notablemente inferior a la existente en otras ciudades españolas. 
También sobresalen en el mapa 2 otras estaciones, como las de Linares-Baeza, Ronda o Lebrija. Esto es consecuencia de la variedad de servicios que prestan, ya que se localizan en ciudades medias que cuentan con amplios ámbitos de influencia y que suelen ser cabeceras comarcales.

La estación de Bobadilla, antiguo nodo de los desplazamientos ferroviarios interiores en Andalucía, mantiene en la actualidad un escaso volumen de viajeros, a pesar de ser el vínculo de numerosos transbordos. La reorganización de los enlaces ferroviarios entre ciudades le ha conducido a una pérdida paulatina de usuarios, ya que los transbordos son menores y suelen repartirse por la geografía andaluza. Además, la construcción de las nuevas líneas de alta velocidad potenciará su desuso para el transporte de viajeros, trasladándose los transbordos de estos servicios a la cercana estación de Antequera-Santa Ana.

\section{HACIA UNA MODELIZACIÓN DE UNA RED FERROVIARIA REGIONAL}

En este estudio también se ha planteado, como instrumento para mejorar la penetración que tiene la red ferroviaria andaluza en su territorio, una modelización en la que se han tenido en cuenta tanto las líneas actuales como las planificadas, así como los corredores en estudio. Estos trazados se han integrado en una red única, sin matizar las tipologías de las infraestructuras actuales, ya que se presuponen características similares. El obviar las diversas características vinculadas se debe a la premisa de acondicionar las infraestructuras existentes antes de construir otras secundarias.

Además, es ineludible que las líneas propuestas pasen por núcleos de un tamaño medio, para favorecer su uso y crear alternativas a los viajes por carretera en localidades en las que existe un gran flujo de viajeros. Por ello, se ha tenido en cuenta la disposición geográfica de los núcleos más poblados de la región, así como de aquéllos que tienen un ámbito de influencia más extenso. Éste es un factor esencial para la localización de todo tipo de servicios. En cualquier caso es importante indicar cómo los trazados reflejados son propositivos, correspondiéndose con antiguos corredores clausurados (Alcalá de Guadaíra-Mairena del Alcor-El Viso del Alcor-Carmona; Sanlúcar de Barrameda-Chipiona-Rota; y Puente Genil-Lucena-Cabra-Baena-Martos-Jaén), según se ve en el posterior mapa 3, pero que en la actualidad afectan a ámbitos en los que existe una elevada demanda potencial.

Para conocer la población que se vería directamente beneficiada por las líneas propuestas y planificadas se han estudiado las áreas de influencia que se formarían alrededor de las nuevas infraestructuras. Para ello, se ha considerado, con ayuda del Sistema de Información Geográfica ArcGIS y su extensión Network Analyst, la población de núcleos que se encontrarían a menos de 5, 10 y 15 minutos por carretera ${ }^{1}$.

La construcción de las líneas planificadas y en estudio añadiría 681.891 usuarios potenciales a menos de 5 minutos de una estación de ferrocarril, llegando a 2.363.140 si se amplía la isocrona a 15 minutos (tabla 4). Asimismo, la recuperación de los ejes propuestos permitiría que 281.823 habitantes más se encontrasen a menos de 5 minutos, llegando a afectar a 1.380 .904 personas en un ámbito inferior a los 15 minutos.

La hipotética construcción de estas líneas, y el mantenimiento del entramado actual, favorecería que cerca del $60 \%$ de la población andaluza estuviese a menos de 5 minutos de una estación de ferrocarril, un $73 \%$ a menos de 10 minutos, y a menos de 15 minutos un 82,5\%. La ejecución de estas infraestructuras permitiría que pocas ciudades de importancia quedasen fuera del entramado ferroviario regional, destacando sólo Écija, como se observa en el mapa 3.

A nivel provincial, la plasmación de estas infraestructuras en el territorio supondría un incremento bastante importante de las áreas de influencia, según se aprecia en la tabla 5. Exceptuando Jaén, el resto de provincias estarían por encima del $70 \%$ de su población la que se encontraría dentro de los referidos ámbitos de atracción, llegando a un 93\% en el caso de Sevilla.

\footnotetext{
1 Cabe señalar cómo las cifras pueden variar ligeramente debido a las divergencias entre la toponimia del Nomenclátor del INE y la cobertura utilizada del Instituto de Estadística y Cartografía de Andalucía (IECA).
} 


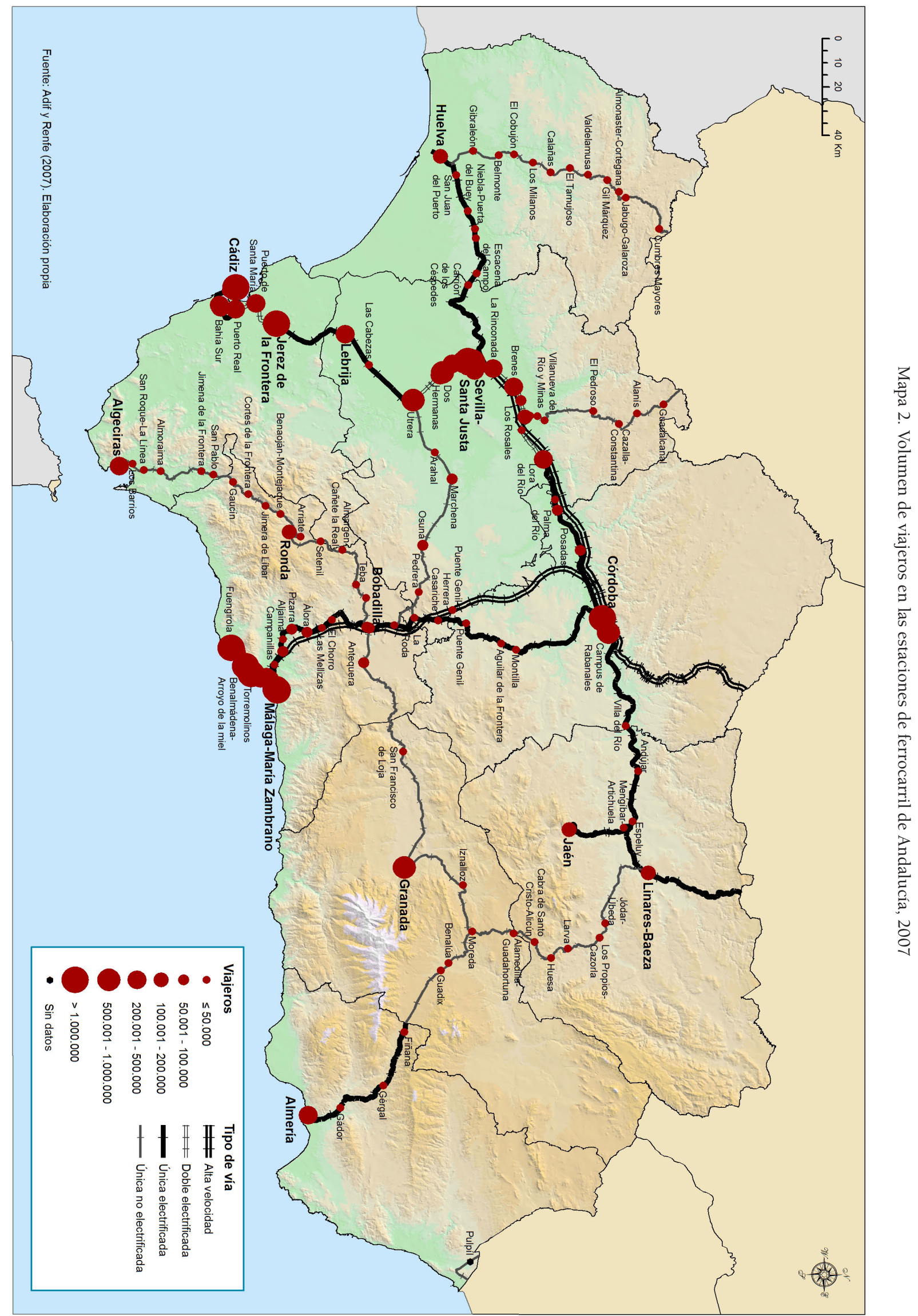


Tabla 4. Población incluida dentro de las áreas de influencia en la red ferroviaria andaluza.

\begin{tabular}{|c|c|c|c|c|c|c|}
\hline \multirow{2}{*}{ Provincia } & \multicolumn{2}{|c|}{$<5$ minutos } & \multicolumn{2}{|c|}{$<10$ minutos } & \multicolumn{2}{|c|}{$<15$ minutos } \\
\hline & Habitantes & $\%^{a}$ & Habitantes & $\%{ }^{a}$ & Habitantes & $\%$ \\
\hline Red actual & 3.811 .984 & 45,9 & 4.662 .813 & 56,2 & 5.522 .176 & 66,5 \\
\hline $\begin{array}{l}\text { Trazados propuestos } \\
\text { en los planes }\end{array}$ & 681.891 & 8,2 & 1.265 .815 & 15,2 & 2.363 .140 & 28,5 \\
\hline $\begin{array}{c}\text { Trazados propuestos } \\
\text { en el estudio }\end{array}$ & 281.823 & 3,4 & 1.182 .909 & 14,2 & 1.380 .904 & 16,6 \\
\hline Total & 4.931 .394 & 59,4 & 6.050 .635 & 72,9 & 6.851 .559 & 82,5 \\
\hline
\end{tabular}

Fuente: Instituto de Estadística y Cartografía de Andalucía, 2010. Elaboración propia

Éste es el caso de Almería, que pasaría de atender a un 26\% de la población a menos de 5 minutos, a un $52 \%$; y a un $72 \%$ a menos de 15 minutos. La provincia de Málaga lo incrementaría hasta un $88 \%$ a menos de 15 minutos, acercándose a la proporción existente en Sevilla. Por el contrario, en Jaén se produciría un incremento leve, que llegaría a los 5 puntos para la población que se encuentra a menos de 15 minutos.

La extensión incluida dentro de las áreas de influencia también aumentaría, aunque en menor medida, si se compara con la población que se podría beneficiar. Esto se debe a que los trazados planificados o propuestos pretenden transcurrir por espacios donde se localizan ciudades medias de un tamaño considerable.

No obstante, la extensión de las áreas de influencia llega en algunos casos a casi triplicar los valores actuales, como en Almería, donde pasa de un 10,6\% a un 30,5\%. Málaga cuenta con el ámbito de influencia más amplio, ya que abarcaría el 62,3\% de la provincia. A nivel regional, la superficie a menos de 5 minutos pasaría de un $5,4 \%$ a un $7,6 \%$, a menos de 10 minutos de un $17,2 \%$ a un $24 \%$, y a menos de 15 minutos de un $29,5 \%$ a un $39,2 \%$ (tabla 6 ).

Tabla 5. Población incluida a nivel provincial dentro de las áreas de influencia de la red ferroviaria propuesta, 2009.

\begin{tabular}{|c|c|c|c|c|c|c|c|c|c|}
\hline \multirow[b]{2}{*}{ Provincia } & \multicolumn{3}{|c|}{$<5$ minutos } & \multicolumn{3}{|c|}{$<10$ minutos } & \multicolumn{3}{|c|}{$<15$ minutos } \\
\hline & Hab. & $\% *$ & $\begin{array}{c}\text { Red } \\
\text { actual } \\
(\%)\end{array}$ & Hab. & $\% *$ & $\begin{array}{c}\text { Red } \\
\text { actual } \\
(\%)\end{array}$ & Hab. & $\% *$ & $\begin{array}{c}\text { Red } \\
\text { actual } \\
(\%)\end{array}$ \\
\hline Almería & 354.918 & 51,9 & 26,2 & 457.832 & 66,9 & 33,3 & 491.816 & 71,9 & 38,6 \\
\hline Cádiz & 643.238 & 52,3 & 43,1 & 817.430 & 66,4 & 54,5 & 961.273 & 78,1 & 66,6 \\
\hline Córdoba & 459.161 & 57,1 & 47,8 & 501.546 & 62,4 & 51,1 & 606.778 & 75,5 & 61,0 \\
\hline Granada & 447.789 & 49,3 & 31,0 & 616.672 & 68,0 & 35,2 & 719.380 & 79,3 & 46,3 \\
\hline Huelva & 268.377 & 52,3 & 44,4 & 323.327 & 63,0 & 53,4 & 425.392 & 82,9 & 65,9 \\
\hline Jaén & 186.549 & 27,9 & 24,4 & 328.864 & 49,1 & 42,5 & 443.837 & 66,3 & 61,2 \\
\hline Málaga & 1.160 .592 & 72,9 & 57,5 & 1.322 .205 & 83,0 & 62,1 & 1.401 .622 & 88,0 & 67,8 \\
\hline Sevilla & 1.410 .666 & 74,2 & 59,4 & 1.682 .655 & 88,6 & 78,1 & 1.771 .633 & 93,2 & 89,5 \\
\hline Andalucía & 4.931 .394 & 59,4 & 45,9 & 6.050 .635 & 72,9 & 56,2 & 6.851 .559 & 82,5 & 66,5 \\
\hline
\end{tabular}

Fuente: Instituto de Estadística y Cartografía de Andalucía, 2010. Elaboración propia 


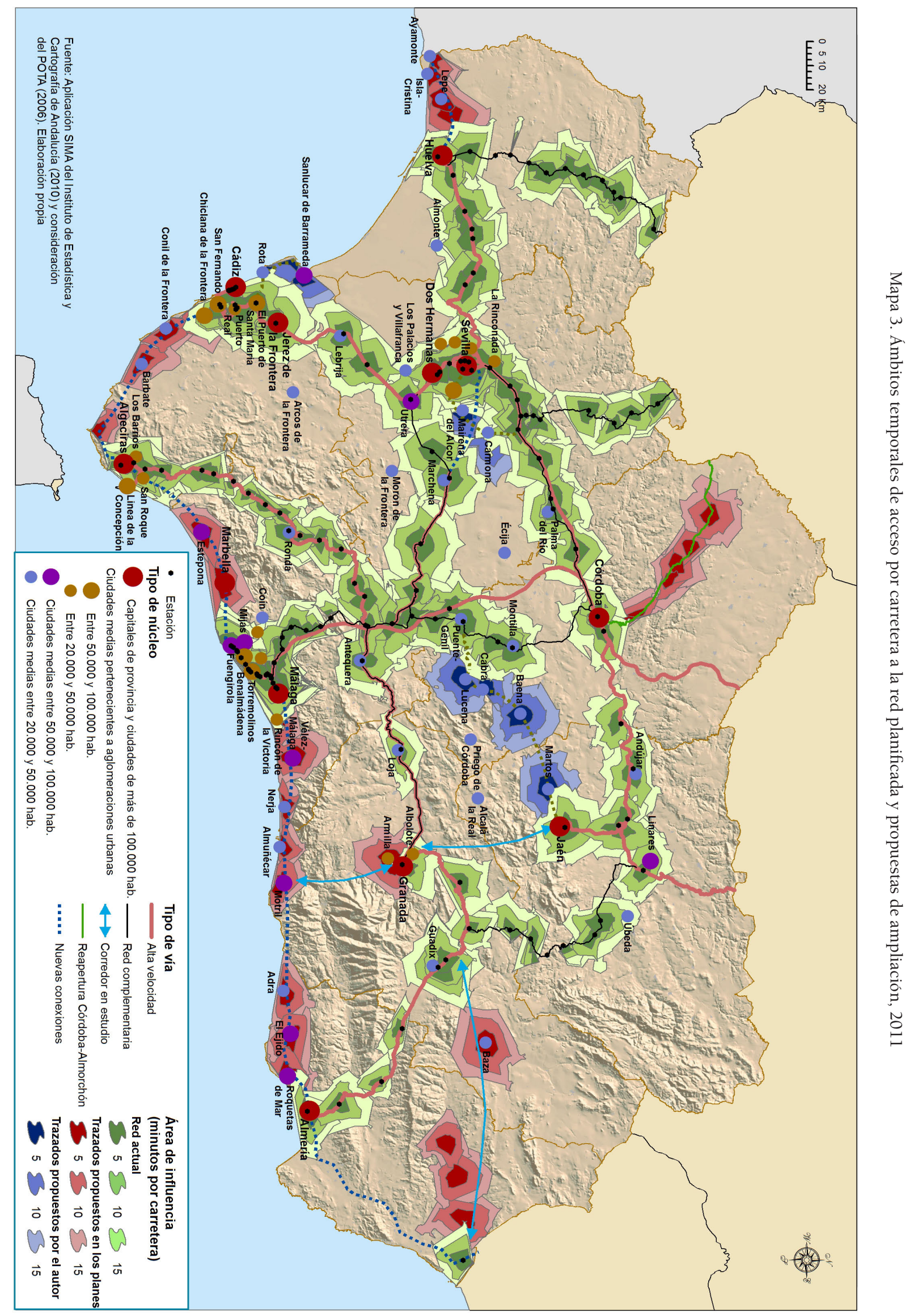


Tabla 6. Extensión incluida a nivel provincial dentro de las áreas de influencia de la red ferroviaria propuesta $(\mathrm{km} 2), 2009$.

\begin{tabular}{|c|c|c|c|c|c|c|c|c|c|}
\hline \multirow[b]{2}{*}{ Provincia } & \multicolumn{3}{|c|}{$<5$ minutos } & \multicolumn{3}{|c|}{$<10$ minutos } & \multicolumn{3}{|c|}{$<15$ minutos } \\
\hline & Ext. & $\% *$ & $\begin{array}{c}\text { Red } \\
\text { actual } \\
(\%)^{*}\end{array}$ & Ext. & $\% *$ & $\begin{array}{l}\text { Red actual } \\
(\%)^{*}\end{array}$ & Ext. & $\% *$ & $\begin{array}{c}\text { Red } \\
\text { actual } \\
(\%)^{*}\end{array}$ \\
\hline Almería & 438 & 5,0 & 1,3 & 1.612 & 18,4 & 5,2 & 2.678 & 30,5 & 10,6 \\
\hline Cádiz & 709 & 9,5 & 6,3 & 2.051 & 27,6 & 18,1 & 3.070 & 41,2 & 30,4 \\
\hline Córdoba & 790 & 5,7 & 3,0 & 2.864 & 20,8 & 11,3 & 5.097 & 37,0 & 21,2 \\
\hline Granada & 457 & 3,6 & 1,9 & 1.809 & 14,3 & 8,2 & 3.653 & 28,9 & 17,7 \\
\hline Huelva & 830 & 8,2 & 6,6 & 2.369 & 23,3 & 19,5 & 3.729 & 36,7 & 31,8 \\
\hline Jaén & 496 & 3,7 & 3,2 & 1.967 & 14,6 & 12,6 & 3.519 & 26,1 & 22,8 \\
\hline Málaga & 1.017 & 13,9 & 11,2 & 2.937 & 40,2 & 31,9 & 4.550 & 62,3 & 49,4 \\
\hline Sevilla & 1.952 & 13,9 & 11,0 & 5.430 & 38,7 & 33,3 & 8.010 & 57,0 & 53,8 \\
\hline Andalucía & 6.688 & 7,6 & 5,4 & 21.040 & 24,0 & 17,2 & 34.304 & 39,2 & 29,5 \\
\hline
\end{tabular}

Fuente: Instituto de Estadística y Cartografía de Andalucía, 2010. Elaboración propia

\section{PROPUESTAS DE ACTUACIÓN PARA LA RED FERROVIARIA ANDALUZA}

La potenciación de ciertos servicios de transporte ferroviario permite diversificar las opciones para desplazarse, mejorando las condiciones para acceder a bienes y servicios. Del mismo modo, suelen generarse otro tipo de consecuencias indirectas, como es el aumento de la actividad económica en las ciudades donde realiza parada, como sucede con las estaciones que prestan servicios de alta velocidad (De Rus Mendoza y Román, 2006).

Esta dinámica se genera si se integra el proyecto en el territorio y existen otros factores que influyan en el desarrollo socioeconómico, puesto que por sí solos los efectos son mínimos (Bellet, Alonso, y Casellas, 2010). Su impulso debería basarse en la existencia de paradas en las grandes ciudades andaluzas e intermedias en localidades donde exista una demanda potencial que pueda extenderse a sus respectivas comarcas.

Sin embargo, la transformación programada de parte de la red ferroviaria en trazados de alta velocidad provocará una previsible reducción de las paradas intermedias, mientras que los nodos de estas redes se beneficiarán de una considerable mejora en los desplazamientos. Estas pautas siguen vigentes en la red andaluza a pesar de su elevado coste económico y ambiental. Además, el tren de alta velocidad ofrece poco beneficio con respecto al convencional mejorado para trayectos inferiores a 150-200 kilómetros (SDG, 2004), distancia que aparece en muchos de los trazados planificados en Andalucía.

No obstante, la eclosión de la crisis económica iniciada en 2008 está fomentando la posible reconsideración de la política en materia ferroviaria llevada a cabo en años anteriores, con el fin de analizar la idoneidad de continuar con el modelo aplicado en el periodo de expansión económica. Los principales cambios que se pueden introducir son una mayor utilización e incremento en la calidad de las infraestructuras existentes, así como el fomento de las relaciones entre ambas tipologías.

De todas maneras, cabe señalar cómo el modelo seguido de desarrollo ferroviario ha propiciado una serie de beneficios en las localidades donde realizan parada. Éstos son principalmente el ahorro en los tiempos de viaje, su mayor capacidad de carga, la reducción de externalidades con respecto a otros medios, el tráfico que genera, la posibilidad de originar commuters, la contracción del espacio, o la calidad 
del transporte. A pesar de presuponerse estos efectos, no se deben valorar todas las consecuencias hasta pasado unos años, ya que a largo plazo se pueden modificar los sistemas de transporte y la Ordenación del Territorio como consecuencia de la nueva infraestructura (Bellet, Alonso y Casellas, 2010).

Por todo lo indicado con anterioridad, es interesante reflexionar sobre si se están creando dos grupos de habitantes en Andalucía, los que se benefician de estas inversiones y aquéllos que no, ya que su funcionamiento pueda provocar la supresión o disminución de los servicios ferroviarios actuales. A continuación, se señalan los principales efectos negativos, no por conocidos menos evidentes, de la excesiva potenciación de la alta velocidad sobre el ferrocarril convencional.

- Elitismo: el coste elevado de la construcción de la infraestructura se traslada al precio del billete, excluyendo a las personas que tienen un menor poder adquisitivo. Además, la implantación de la red AVE suele acarrear una drástica reducción o el cese de los viajes comerciales de la red convencional, provocando que los usuarios más humildes no puedan hacer frente a los nuevos precios. Esto termina potenciando la utilización de medios de transportes más económicos, como el autobús o el vehículo privado.

- Consumo energético: las altas velocidades que alcanzan los trenes suelen acompañarse de un elevado gasto energético durante su construcción y funcionamiento, siendo sólo superado por el transporte aéreo (Estevan Estevan y Sanz Alduán, 1994).

- Impacto ambiental: éste proviene del montaje de las infraestructuras, debido a la creación de enormes puentes, túneles y curvas. La construcción de estos elementos supone la modificación y ocupación de grandes extensiones y el movimiento de millones de metros cúbicos de tierra. Además, su funcionamiento provoca diversos impactos, como el efecto barrera para la fauna del entorno.

- Desvertebración del territorio: esta tipología ferroviaria, al estar diseñada para conectar a poblaciones distantes, provoca la exclusión de las ciudades intermedias. Su construcción, y el posterior abandono de la red convencional, origina la articulación territorial, al perderse las paradas que realizaban los trenes de media distancia y larga distancia. La inversión en este tipo de infraestructuras termina potenciando los nodos que cuentan con mayor peso económico, disminuyendo su alcance en los secundarios al quedarse fuera de las mejoras ferroviarias.

- Efectos económicos: la construcción de una línea de alta velocidad genera un impacto económico positivo en muchos sectores de las ciudades beneficiadas. El turismo puede ser uno de estos sectores, ya que se incrementa el número de visitantes, aunque se reducen las pernoctaciones. No obstante, en el turismo de negocios y conferencias se suele notar más la disminución de las pernoctaciones, al ser más fácil retornar a las localidades de origen (Albalate y Bel, 2010). Del mismo modo, en las ciudades cuyas condiciones económicas son más débiles puede dar lugar a que las actividades se desplazasen al nodo con mayor potencial.

- Ruido: Su funcionamiento genera una elevada contaminación acústica, siendo el efecto que más aprecia la población y la fauna cercana (Vázquez Espí, 2003). La exposición continuada puede provocar diversos problemas de salud.

Frente a este modelo de alta velocidad, que es el seguido en la mayoría de las líneas, existe la posibilidad de extender el patrón utilizado en el trazado que conecta Sevilla con Cádiz. Las actuaciones llevadas a cabo consisten principalmente en la remodelación de las infraestructuras actuales, ejecutándose una duplicación de la vía en ancho ibérico mejorado, con la posibilidad de cambiarse a internacional si fuese necesario en un futuro.

La construcción con estas características permitirá el paso de ferrocarriles a velocidades que rondarán los $200 \mathrm{~km} / \mathrm{h}$. Asimismo, se podrán compatibilizar las infraestructuras con trenes de larga distancia, media distancia y cercanías, así como la inclusión de parte del tranvía que discurrirá por la Bahía de Cádiz. Esto permitiría crear una infraestructura polivalente, en la que se ofrezcan servicios de calidad entre las localidades beneficiarias. En la tabla 7 se exponen las principales diferencias de los modelos existentes de alta velocidad en Andalucía. 
Tabla 7. Modelos imperantes de alta velocidad

\begin{tabular}{|c|c|}
\hline Ancho internacional & Ancho ibérico (Línea Sevilla-Cádiz) \\
\hline $\begin{array}{c}\text { Impacto ambiental generado por la construcción de un nuevo } \\
\text { trazado que no permite grandes curvas, multiplicando el } \\
\text { emplazamiento de puentes y túneles }\end{array}$ & $\begin{array}{c}\text { Reutilización y mejora de la red actual, evitando el impacto que } \\
\text { genera la ocupación del suelo por una nueva infraestructura }\end{array}$ \\
\hline $\begin{array}{c}\text { Desvertebración territorial por las escasas paradas que realiza en en } \\
\text { las ciudades medias }\end{array}$ & $\begin{array}{c}\text { Posibilidad de atender a más habitantes, ya que pueden realizarse } \\
\text { paradas en más ciudades intermedias durante el recorrido }\end{array}$ \\
\hline $\begin{array}{c}\text { Menor correlación con la red convencional debido a los diferentes } \\
\text { anchos de vía, lo que dificulta la prestación de servicios de } \\
\text { cercanías y mercancías }\end{array}$ & $\begin{array}{c}\text { Utilización polivalente del trazado, pudiéndose prestar, sobre las } \\
\text { mismas infraestructuras, servicios de alta velocidad, cercanías, } \\
\text { tranviarios y de mercancías }\end{array}$ \\
\hline $\begin{array}{c}\text { Mayor coste del billete para amortizar la construcción de una } \\
\text { nueva infraestructura }\end{array}$ & $\begin{array}{c}\text { Precios competitivos con respecto a autobús y automóvil debido a } \\
\text { la alta utilización de la infraestructura y al ser menores los costes } \\
\text { de construcción, mantenimiento y funcionamiento }\end{array}$ \\
\hline
\end{tabular}

Elaboración propia

Por tanto, se pueden resumir finalmente aquellos aspectos que merecen ser implementados para mejorar la política ferroviaria en Andalucía en beneficio del desarrollo socioeconómico regional, de una mejor vertebración territorial, y de la reducción del impacto ambiental generado por la movilidad que realizan sus ciudadanos. En concreto, se proponen las siguientes actuaciones:

- Ampliar e integrar nuevas ciudades medias en el entramado ferroviario andaluz, como Carmona, Motril o Ayamonte.

- Mejorar las prestaciones de los servicios ferroviarios convencionales de media distancia, en especial en el Eje Ferroviario Transversal de Andalucía (EFTA). para reducir los tiempos de viaje e incrementar su calidad.

- Plantear los nuevos trazados con estaciones más cercanas a los núcleos de población, para favorecer así una mayor utilización de estas infraestructuras, y evitar casos como el de la estación de AVE en Puente Genil-Herrera.

- Conectar las capitales provinciales con las localidades más pobladas de su entorno, de manera que se generen servicios que articulen mejor el territorio y potencien el uso del ferrocarril frente al automóvil privado, según se plantea para la nueva red de cercanías de Sevilla.

- Establecer tarifas y servicios más atractivos para los usuarios que cuentan con menor poder adquisitivo.

- Extender y/o crear nuevas redes de transporte metropolitano, según está previsto para las ciudades de Córdoba o Granada.

- Favorecer la accesibilidad de personas con movilidad reducida.

- Incrementar la intermodalidad a partir de la optimización y construcción de nuevos espacios en los que realizar los intercambios de viajeros con otros medios de transporte, como sucede en la estación intermodal de Málaga, donde se reúnen las terminales de tren, autobús y metro.

- Crear más plazas de aparcamientos disuasorios en los aledaños de las estaciones de ferrocarril para reducir el uso del automóvil.

\section{CONCLUSIONES}

Las diferentes intervenciones realizadas y previstas encumbran al transporte ferroviario como alternativa originaria del pasado pero aplicable a los problemas actuales de movilidad, contando entre sus principales características con la rapidez, el menor coste, la seguridad y el respeto por el entorno. El cambio de mentalidad, tanto social como política, se está trasladando a la red andaluza, donde en pocos años, y según la planificación establecida, debería ser una realidad la interacción de diferentes modos ferroviarios (tranvía, metro, ferrocarril convencional y alta velocidad). 
La integración de la alta velocidad en la red ferroviaria andaluza puede contribuir al desarrollo territorial siempre que se complemente con unos servicios convencionales de mayor calidad, ya que permitiría el acercamiento entre ciudades alejadas. Su ejecución debería pasar por el previo desdoblamiento de aquellas líneas convencionales que, una vez mejoradas, mantengan un tráfico elevado que impida la prestación de servicios adecuados a las demandas de la población, así como para largas distancias en las que las diferencias de tiempo puedan ser considerables.

En los trayectos medios y cortos la inclusión de la alta velocidad no tiene una elevada utilidad, puesto que no se produce una reducción considerable en los tiempos de viaje con respecto al ferrocarril convencional mejorado. Además, la adecuación de los medios existentes posibilitaría una mayor vertebración del territorio, al mantenerse las estaciones actuales, y su papel dentro de las tramas urbana, pudiéndose compatibilizar con el transporte de mercancías que se pretende impulsar, como ocurre para la línea Sevilla-Cádiz.

Del mismo modo, la potenciación de una movilidad urbana e interurbana más sostenible entre poblaciones vecinas puede trasladarse a una intermodalidad real que integre todos los medios de transporte, en la que ir a pie o en bicicleta sea importante en los desplazamientos, completándose principalmente con los diferentes modos ferroviarios y por los autobuses públicos. En la actualidad, en las grandes ciudades, se están creando espacios intermodales, localizándose diferentes medios de transporte en ámbitos cercanos, con el fin de facilitar el intercambio de viajeros. Falta, sin embargo, un impulso contundente en las ciudades medias que facilite, entre otros, la extensión de las áreas de influencia de las estaciones ferroviarias de viajeros más allá del ámbito local, cuestión que con los planteamientos aquí propuestos podría alcanzar hasta el $82,5 \%$ de la población andaluza con residencia a menos de 15 minutos en coche de una de ellas.

El principal problema con el que se encuentran las diferentes propuestas de mejora y ampliación de la red regional es la actual crisis económica. En los últimos años se había generalizado la necesidad de dotar a las grandes ciudades de entramados de transporte ferroviario que canalizasen la movilidad cotidiana de la población, así como la adecuación de las redes interurbanas, para potenciar unos desplazamientos medios y largos más sostenibles, pero la situación presente puede suponer su retraso o cancelación, debido a la falta de financiación.

No obstante, en la actualidad, es el mejor momento para potenciar el uso del transporte público, ya que su menor coste lo hace más asequible para las familias que se encuentran en una situación de mayor vulnerabilidad. Oferta que debe de ser atractiva también para la población en general. Por ello, se deben impulsar cambios que permitan ajustar los servicios ferroviarios tanto en coste como en calidad a las necesidades de los potenciales demandantes.

En definitiva, se puede señalar cómo el ferrocarril se ha transformado en una alternativa rescatada del pasado para los problemas de movilidad que afecta en nuestros días a la población, lo que debe facilitar que los próximos años sean previsiblemente los más prolíferos del ferrocarril, dejando atrás la conformación vertiginosa y desordenada de la red que la caracterizó durante el siglo XIX. Un desarrollo equilibrado, y que vertebre la comunidad autónoma de Andalucía, con servicios tanto convencionales como de alta velocidad, serán los principales factores que influyan en su éxito. Por el contrario si su evolución no es equilibrada, dentro de unos años sólo existirían servicios de alta velocidad y metropolitanos, focalizados en los centros regionales y en las ciudades litorales; mientras que el resto de la región se encontraría desconectada de la red, lo que induciría el aumento de los desplazamientos motorizados privados dentro de la Comunidad andaluza, así como a un menor desarrollo socioeconómico de estas localidades, al tener menos alternativas para el desplazamiento de sus propios habitantes. 


\section{BIBLIOGRAFÍA}

ALBALATE, D. y BEL, G. (2010): "High-Speed Rail: Lessons for Policy Makers from Experiences Abroad". Working Paper. Research Institute of Applied Economics.

AYCART LUENGO, C. (2001): "Vías Verdes, reutilización de ferrocarriles en desuso para movilidad sostenible, ocio y turismo", en Informes de la Construcción Vol. 53 n 475, pp. 17-29.

BELLET, C., ALONSO, P. y CASELLAS, A. (2010): "Infraestructuras de transporte y territorio. Los efectos estructurantes de la llegada del tren de Alta Velocidad en España”. Boletín de la Asociación de Geógrafos Españoles, $n^{\circ}$ 52, pp. 143-163.

CANO GARCÍA, G. (dir. y coord.) (2002): Las Comarcas Andaluzas. Sevilla. Ediciones Tartessos.

CARBONELl ROMERO, A. (1990): "Los ferrocarriles", en Carbonell Romero y otro Las infraestructuras en España: carencias y soluciones. Madrid, Instituto de Estudios Económicos.

CONSEJERÍA DE OBRAS PÚBLICAS Y TRANSPORTES (1988): Plan Estratégico Ferroviario de Andalucía. Sevilla, Dirección General de Transportes de la Junta de Andalucía.

CONSEJERÍA DE OBRAS PÚBLICAS Y TRANSPORTES (2006): Plan de Ordenación del Territorio de Andalucía (POTA). Sevilla, Junta de Andalucía.

CONSEJERÍA DE OBRAS PÚBLICAS Y TRANSPORTES (2008): Plan de Infraestructuras para la Sostenibilidad del Transporte en Andalucía (PISTA 2007-2013). Sevilla, Junta de Andalucía.

DE RUS MENDOZA, G. y ROMÁN, C. (2006): "Análisis económico de la línea de Alta Velocidad MadridBarcelona". Revista de Economía Aplicada n 42 (vol. XIV), pp. 35-79.

ESTEVAN ESTEVAN, A y SANZ ALDUÁN, A. (1994): Hacia la reconversión ecológica del transporte en España. Madrid, Fundación Hogar del Empleado/Centro de Investigación para la Paz, s.f.

GAVIRA NARVÁEZ, A. (2008): "Perspectivas para el Desarrollo Territorial de las Infraestructuras Ferroviarias en la Provincia de Sevilla", en Antón Burgos F. J. y Sánchez Moral, S. (ed.) Comercio, Servicios y Transporte. Patrones de una Sociedad Avanzada. Madrid, Grupo Geografía de los Servicios. Asociación de Geógrafos Españoles, pp. 289-302.

JUNTA DE ANDALUCÍA (2007): Reforma del Estatuto de Autonomía para Andalucía. Sevilla.

LÓPEZ PÉREZ, F. (1990): "El Transporte Ferroviario Andaluz", en Cano García, G. (dir. y coord.) Geografía de Andalucía Tomo VI. Sevilla, Ediciones Tartessos. pp. 147-200.

MINISTERIO DE FOMENTO (2005): Plan Estratégico de Infraestructuras y Transportes 2005-2020. Madrid, Centro de Publicaciones del Ministerio.

MIRALLES-GUASCH, C. (2002): Ciudad y transporte. El binomio imperfecto. Barcelona, Editorial Ariel Geografía.

SEGUÍ PONS, J. M. y MARTÍNEZ REYNÉS, M. R. (2004): Geografía de los Transportes. Palma de Mallorca, Universidad de les Illes Balears.

STEER DAVIES GLEAVE (SDG) (2004): High Speed Rail: International Comparisons, Final report. London, Commission for Integrated Transport.

VALENZUELA MONTES, L. M. (2009): El metro ligero como factor de innovación ambiental en las áreas metropolitanas andaluzas. Sevilla, Centro de Estudios Andaluces de la Consejería de la Presidencia de la Junta de Andalucía.

VÁZQUEZ ESPÍ, M. (2003): Transporte y energía [en línea] <http:/habitat.aq.upm.es/boletin/n28/amvaz.html > [Consulta: 11/04/13].

VEGA GONZÁLEZ, G. (1990): "Etapas de la Construcción del Ferrocarril en Andalucía". Jornadas. La nueva situación ferroviaria y sus efectos regionales en Andalucía. Madrid, Centro de Estudios Territoriales y Urbanos, Consejería de Obras Públicas y Transportes de la Junta de Andalucía y Fundación de los Ferrocarriles Españoles, pp. 29-46. 